\title{
Thermogravimetric Analysis-based Characterization of Suitable Biomass for Alkaline Peroxide Treatment to Obtain Cellulose and Fermentable Sugars
}

\begin{abstract}
Akihiro Hideno *
To characterize a suitable biomass for alkaline peroxide treatment, four types of lignocellulosic biomass (rice straw, two Miscanthus spp., and Japanese cypress) were characterized using thermogravimetric analysis. Before the alkaline peroxide treatment, rice straw had the lowest initial thermal degradation temperature and Japanese cypress had the highest. After alkaline peroxide treatment, this trend was reversed, such that the highest initial thermal degradation temperature was for alkaline-peroxide treated rice straw. Hemicellulose and lignin content significantly affected the thermal degradation behavior and alkaline peroxide treatment efficiency. Among the four lignocelluloses, raw rice straw exhibited the highest reducing capability, whereas the treated rice straw exhibited the lowest reducing capability. Surface morphology and crystallinity indicated that when the rice straw was subjected to AP treatment at room temperature for $10 \mathrm{~min}$, crystalline cellulose microfibrils were exposed and concentrated on the surface. Thus, among the samples tested, rice straw was found to be the most suitable biomass for alkaline peroxide treatment.
\end{abstract}

Keywords: Alkaline peroxide treatment; Rice straw; Thermogravimetric analysis; Reducing capability; Cellulose

Contact information: Paper Industry Innovation Center, Ehime University, 127 Mendori-cho, Shikokuchuo, Ehime 799-0113, Japan Tel: +81-089-622-3230;

*Corresponding author: a-hideno@agr.ehime-u.ac.jp

\section{INTRODUCTION}

Lignocellulose consists primarily of cellulose, hemicellulose, and lignin. It is the main feedstock for biorefinery processes, such as those used for the production of fuels and chemicals derived from plant biomass. To use lignocellulose effectively, its individual constituents require isolation and analysis. Cellulose is the most abundant organic material on Earth (Hon 1994). The optimization of isolation of cellulose from lignocellulose such as pulping process and pretreatment for enzymatic hydrolysis, and analytical techniques are important because cellulose is used in the production of pulp and paper, cellulose nanofibers (Yano 2010; Isogai et al. 2011), and fermentable sugars (Hideno et al. 2013a).

The pulping process for wood material usually includes delignification, partial removal of hemicellulose, and isolation of cellulosic fibers (Kleppe 1970). Kraft pulping is considered a preferred technique because it generates pulp very efficiently, has a high chemical recovery rate, and produces energy by burning black liquor. However, large digesters and highly specialized equipment are required for kraft pulping. Pretreatment for enzymatic hydrolysis of lignocellulose includes a similar pulping process (Mosier et al. 2005). Several studies using pretreatments to obtain cellulose and fermentable sugars from agricultural residues including rice straw and herbaceous biomass such as Miscanthus 
plants have been conducted (Mosier et al. 2005; Hendriks and Zeeman 2008; Hideno et al. $2009,2013 b)$. Other reported pretreatments include milling, grinding, and treatments with sulfuric acid, alkalis, and biological agents such as white-rot fungus (Hendriks and Zeeman 2008; Hideno et al. 2009, 2013b). Alkaline-peroxide (AP) treatment is simple and highly effective at removing lignin and hemicellulose, resulting in a high sugar yield in the enzymatic hydrolysis. Furthermore, AP treatment was shown to be a simple and effective pulping process for rice straw (Hideno 2017). However, the effectiveness of AP treatment is different between biomasses and depends on the biomass type. For example, the optimal AP treatment conditions for rice straw, which is the most abundant agricultural waste in Asia (Kim and Dale 2004) were of short duration at approximately $10 \mathrm{~min}$ (short-term) and at room temperature (approximately $25^{\circ} \mathrm{C}$ ) (Hideno 2017). Nevertheless, other types of biomass required over $12 \mathrm{~h}$ and $70{ }^{\circ} \mathrm{C}$ for optimal AP treatment (Hideno 2013b,c). Therefore, AP treatment can efficiently and rapidly remove lignin and hemicellulose from rice straw without requiring any specialized equipment or reactors. Although the physical properties of AP-treated rice straw are not well known, there is evidence that high enzymatic digestion results in AP-treated rice straw containing a small amount of lignin (Hideno 2017).

Thermogravimetric analysis (TGA) has been suggested for heterogeneous organic material characterization, such as lignocellulosic biomass (Negro et al. 2003). TGA is often used to show thermal degradation kinetics (Antal and Varhegyi 1995). However, TGA has also been suggested as a simple evaluation method to judge effectiveness of pretreatment and pulping conditions (Hideno 2016, 2018). The areas of differential TG (DTG) peaks and fitted DTG curves obtained from TGA are remarkably correlated with the cellulose components and crystallinity. Moreover, the analysis of a DTG curve can detect the damages to hemicellulose and lignin in ball milling (Hideno 2018). However, the thermal degradation properties of AP-treated biomass are not well known.

To show the influences of AP treatment on select biomass and to characterize the AP-treated biomass, the effects of AP treatment on the thermal degradation properties of Japanese cypress, rice straw, and Miscanthus plants were investigated and compared in this study. Specifically, the relationships between thermal degradation properties and processing efficiency of AP treatment were examined. Moreover, rice straw, which is a suitable biomass for AP treatment, was characterized in detail before and after AP treatment.

\section{EXPERIMENTAL}

\section{Materials}

Lignocellulosic biomass

Japanese cypress (Chamaecyparis obtusa), rice straw, and Miscanthus plants ( $M$. sinensis and M. giganteus) were used as described in previous reports (Hideno et al. 2013a,b, 2017). The samples were manually cut to approximately 1 to $2 \mathrm{~cm}$ length and drymilled in a blender (Absolute 3, Osaka Chemical Co. Ltd., Osaka, Japan). The dry-milled sample was mechanically sieved for $30 \mathrm{~min}$. The starting material was graded by size from 125 to $500 \mu \mathrm{m}$. 


\section{Methods}

AP treatment

The AP treatment was carried out as described in earlier reports (Hideno et al. 2013, 2017). Approximately $0.5 \mathrm{~g}$ of a sieved sample, $2 \mathrm{~mL}$ of $5 \mathrm{M} \mathrm{NaOH}$, and $3 \mathrm{~mL}$ of hydrogen peroxide reagent (30 to 33\%) were added to a $200 \mathrm{~mL}$ Erlenmeyer flask and thoroughly mixed with a magnetic stirrer using a Teflon ${ }^{\circledR}$ stirrer bar. For rice straw, the reaction was conducted at approximately $25{ }^{\circ} \mathrm{C}$ for approximately $10 \mathrm{~min}$. For the Japanese cypress and Miscanthus plants, the flask was shaken at $150 \mathrm{rpm}$ at $70{ }^{\circ} \mathrm{C}$ for $72 \mathrm{~h}$ by using a shaker (BioShaker BR-22FH, TAITECH Co., Saitama, Japan). After the reaction, approximately $100 \mathrm{~mL}$ of pure water was added to both flasks. The diluted mixture was then neutralized with 1 to $5 \mathrm{~N} \mathrm{HCl}$ solution, washed with about $100 \mathrm{~mL}$ of water, and freeze-dried by using a freeze dryer (FDU-1200, TOKYO RIKAKIKAI Co., LTD, Tokyo, Japan).

$T G A$

TGA of the original and ball milled Miscanthus plants was conducted as described in a previous report (Hideno 2016). Each sample (approximately $5 \mathrm{mg}$ ) was formed into a tablet $(\Phi 4.5 \mathrm{~mm})$ with a hand press. A TGA instrument (TG/DTA6200, Seiko Instruments Inc., Chiba, Japan) was used under a nitrogen atmosphere at a flow rate of $100 \mathrm{~mL} \mathrm{~min}^{-1}$, as described in an earlier report (Uetani et al. 2014). Operating conditions were: RT-110 ${ }^{\circ} \mathrm{C}\left(40{ }^{\circ} \mathrm{C} \mathrm{min}{ }^{-1}\right), 110{ }^{\circ} \mathrm{C}$ for $10 \mathrm{~min}, 110{ }^{\circ} \mathrm{C}$ to $550{ }^{\circ} \mathrm{C}\left(10{ }^{\circ} \mathrm{C} \mathrm{min}{ }^{-1}\right)$, and $550{ }^{\circ} \mathrm{C}$ for 10 min. Differential thermogravimetric (DTG) and relative DTG curves were calculated using Eqs. 1, 2, and 3,

$\mathrm{TG}(\%)=($ weight loss due to thermal decomposition / original weight $) \times 100$

DTG $(\% / \mathrm{min})=\mathrm{TG}(\%) /$ time for increase in temperature $(\mathrm{min})$

Relative DTG = DTG value / maximum DTG value (DTG curve peak)

The sample weight at $120^{\circ} \mathrm{C}$ was defined as the $100 \%$ dry weight.

Curve-fitting for the DTG peak separation was conducted with TA7000 version 10.41 (Hitachi High-Tech Science Co., Tokyo, Japan) and Fityk version 0.9.4 (Fityk, Warsaw, Poland). A split Gaussian method and a Levenberg-Marquardt algorithm were used for peak separation and DTG curve fitting, respectively.

The ratio of delignification and cellulose accumulation in AP treatment

The ratio was calculated using Eqs. 4 and 5 based on the analytical data in the published reports (Hideno et al. 2013a,b; Hideno 2016, 2017) that were summarized in Table 2.

$$
\begin{aligned}
& \text { Delignification }=(a-b) / a \\
& \text { Cellulose accumulation }=d / c
\end{aligned}
$$

In Eqs. 4 and 5, $a$ is lignin content in a raw sample, $b$ is lignin content after AP treatment, $c$ is cellulose content in a raw sample, and $d$ is cellulose content after AP treatment

\section{Analysis of reducing capability}

The number of moles of reducing-end glucose residue/g sample was determined with a modified 2,2'-bicinchoninate (BCA) method (Zhang and Lynd 2005). Each sample was mixed with $800 \mu \mathrm{L}$ of pure water and added to $800 \mu \mathrm{L}$ of BCA reagent. The mixture was incubated at $75{ }^{\circ} \mathrm{C}$ for $30 \mathrm{~min}$ and then centrifuged at approximately $20,000 \times \mathrm{g}$ for 1 
min. The supernatant was analyzed using a spectrophotometer (U-1900, Hitachi HighTechnologies Corporation, Tokyo, Japan) at $\lambda=560 \mathrm{~nm}$. The standard curve was plotted by using a glucose as reference. The absorbance was converted to reducing-end glucose units $(\mathrm{mmol} / \mathrm{g})$. This value also represented the reducing capability because the BCA reagent can react with other reducing materials.

\section{Field-emission scanning electron microscopy (FE-SEM)}

Sample preparation and FE-SEM analysis were conducted as described in a previous report (Hideno et al. 2014). The sample was filtered and laid out as wet sheets, and the water in the sample was replaced with tert-butyl alcohol. The solvent-exchanged sheets were then freeze-dried by using a freeze dryer (FDU-1200, TOKYO RIKAKIKAI Co., LTD, Tokyo, Japan), coated with platinum (Pt) using an ion-sputtering apparatus (JFC-1600, JEOL Ltd., Akishima, Japan) and observed using FE-SEM (JSM-7800F, JEOL Ltd, Akishima, Japan). The accelerating voltages and working distances were 1.5 to $5 \mathrm{kV}$ and 8 to $10 \mathrm{~mm}$, respectively.

\section{RESULTS AND DISCUSSION}

\section{Comparison of Thermal Degradation Behaviors of Raw and AP-treated Biomass}

Relative DTG curves for raw rice straw (raw RS), M. giganteus (raw MG), $M$. sinensis (raw MS), and Japanese cypress (raw JC) were compared (Fig. 1). Rice straw had the lowest thermal degradation temperatures, whereas Japanese cypress had the highest. For example, the temperatures for the four samples at $1 \%$ weight loss were $180.6^{\circ} \mathrm{C}$ (raw RS), $209.6{ }^{\circ} \mathrm{C}$ (raw MS), $225.5{ }^{\circ} \mathrm{C}$ (raw MG), and $232.3^{\circ} \mathrm{C}$ (raw JC) (Table 1). Thermal degradation temperatures less than $200{ }^{\circ} \mathrm{C}$ are affected by non-cellulosic materials such as hemicellulose. The main constituents in the four samples were sugars and lignin components (Hideno et al. 2013a,b; Hideno 2017), as shown in Table 1. Raw RS had a higher arabinose and lower lignin content than the other samples (Table 2). In contrast, raw JC had a higher thermal degradation temperature and high mannose and lignin contents. Thermal degradation temperatures less than $250{ }^{\circ} \mathrm{C}$ were determined by the hemicellulose and lignin content in the samples. The lignin content of any biomass is the main factor affecting pyrolysis as a higher lignin content impedes decomposition (Burhenne et al. 2013). Lignin also increases the thermal degradation temperature of crystalline cellulose but has no influence on amorphous cellulose (Hilbers et al. 2015). These results also show that raw JC had both a higher lignin content (Table 2) and thermal degradation temperature, whereas raw RS had a comparatively lower lignin content (Table 2) and a lower thermal degradation temperature. This correlation between lignin content and thermal degradation temperature may be consistent with AP treatment.

The thermal decomposition temperatures of raw JC and MG were substantially higher than MS and RS. Raw MG and JC were recalcitrant to pretreatment and enzymatic hydrolysis (Hideno et al. 2013a,b). The opposite trend was observed for the thermal degradation temperatures of the AP treated samples. The thermal degradation temperature of AP treated JC was the lowest, whereas those of AP treated RS and MS were higher than those of the other samples. This result suggests that the cellulose in the AP treated samples accumulated when the less thermal-resistant materials such as hemicellulose were eliminated; thus, the thermal decomposition temperatures increased. The results suggest 
that samples with relatively high degradation temperatures such as raw JC have high tolerances to the AP treatment. The thermal decomposition temperatures of the raw samples with higher thermal degradation temperatures were decreased by AP treatment. In contrast, the thermal decomposition temperatures of raw samples with lower thermal degradation temperatures were increased by the AP treatment. For almost all samples, the AP treatment increased the glucose content derived from cellulose. However, except for the JC, the xylose and arabinose content derived from hemicellulose and lignin considerably decreased in response to the AP treatment (Table 2). The AP treatment degraded nearly all the hemicellulose and lignin because they have low thermal degradation temperatures. In contrast, the treatment concentrated the crystalline cellulose in the herbaceous biomass. The trends in the thermal degradation properties of untreated samples were the opposite of those for the AP-treated samples (Fig. 1). AP treatment increased thermal degradation temperatures for samples that were sensitive to it. Materials with low thermal degradation temperatures were almost entirely eliminated, and the crystalline cellulose was purified. In contrast, the AP treatment reduced the thermal degradation temperatures of samples with low sensitivity to it. These samples retain their constituents with lower thermal degradation temperatures even after AP treatment. The results suggest that thermal analyzes of raw samples can be used to estimate AP treatment efficiency.

(a)

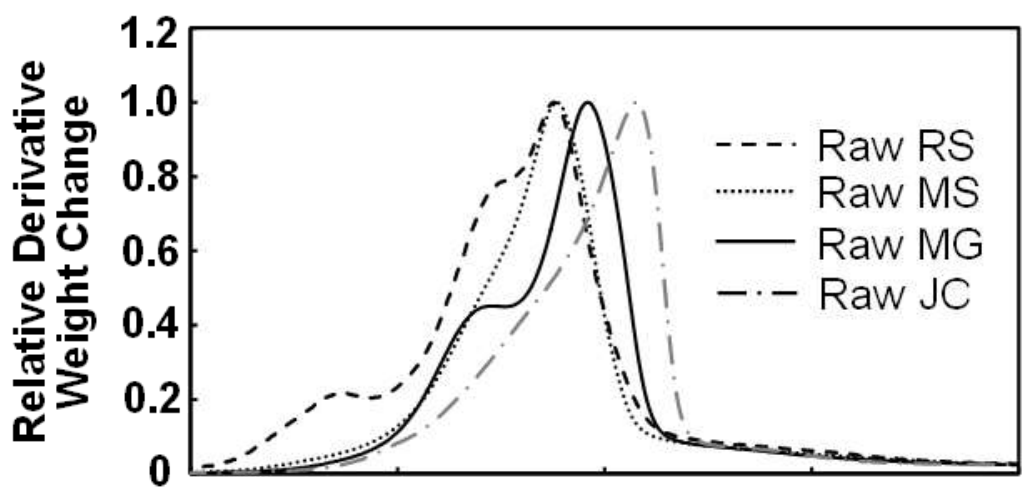

(b)

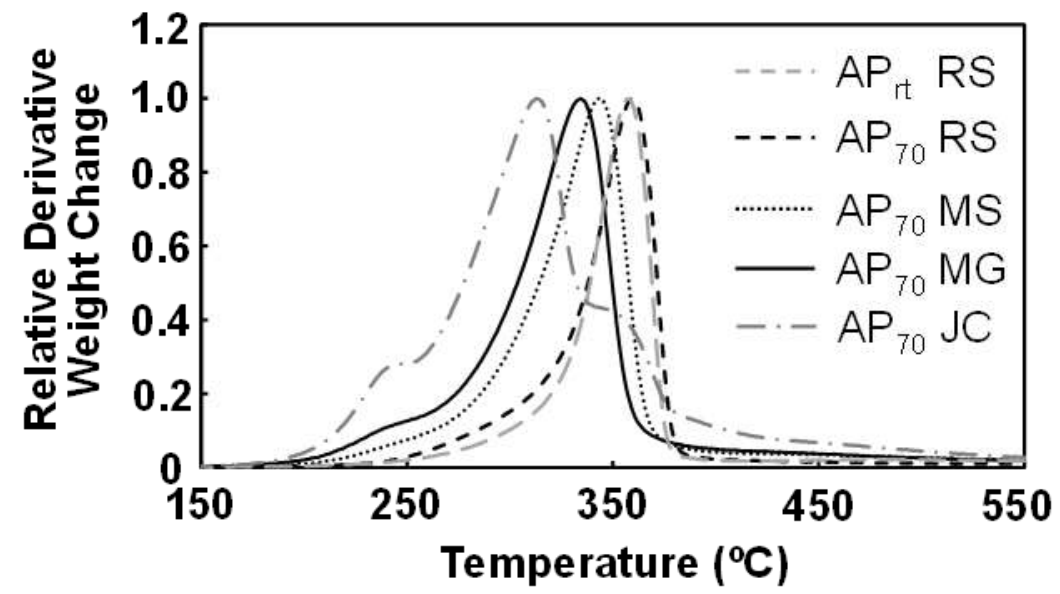

Fig. 1. Derivative thermogravimetric (DTG) curves of (a) raw materials and (b) alkaline peroxidetreated materials

RS: rice straw; MS: M. sinensis; MG: M. giganteus; JC: Japanese cypress; AP70: alkaline peroxide treatment at $70^{\circ} \mathrm{C}$; APrt: alkaline peroxide treatment at approximately $25^{\circ} \mathrm{C}$.

DTG curves of raw MS and MG were used as standards and are cited in a previous report. 
Table 1. Temperatures at the Required Thermal Gravimetric Values (Weight Loss during Thermal Decomposition) and Maximum Differential TG Values in MG, MS, and Rice Straw

\begin{tabular}{|l|c|c|c|c|c|c|}
\hline & \multicolumn{5}{|c|}{ Thermal degradation $\left({ }^{\circ} \mathrm{C}\right)$ at the TG $(\%)$} & $\begin{array}{c}\text { Temperature } \\
\left({ }^{\circ} \mathrm{C}\right) \text { at } \\
\text { DTGmax }\end{array}$ \\
\hline Sample & 99 & 95 & 90 & 85 & 80 & 341.5 \\
\hline Raw MG & 225.5 & 264.0 & 280.1 & 292.3 & 303.3 & 327.2 \\
\hline Raw MS & 209.6 & 258.3 & 278.6 & 290.9 & 300.6 & 325.7 \\
\hline Raw RS & 180.6 & 218.2 & 250.2 & 271.8 & 284.4 & 365.2 \\
\hline AP70-MG & 232.2 & 272.4 & 292.8 & 306.3 & 317.4 & 334.8 \\
\hline AP70-MS & 220.0 & 265.6 & 289.2 & 301.8 & 311.2 & 343.5 \\
\hline AP70-RS & 232.3 & 280.7 & 302.2 & 316.4 & 326.2 & 359.2 \\
\hline APrt-RS & 230.0 & 286.6 & 309.1 & 321.9 & 330.0 & 357.5 \\
\hline AP70-JC & 206.6 & 239.2 & 261.7 & 277.3 & 288.3 & 313.3 \\
\hline
\end{tabular}

Table 2. Comparison of the Main Chemical Components in Raw and Alkaline Peroxide treated Biomass

\begin{tabular}{|c|c|c|c|c|c|c|}
\hline \multirow{2}{*}{ Sample } & \multicolumn{4}{|c|}{ Main Constituent Sugar $(\mathrm{mg} / \mathrm{g})$} & \multirow{2}{*}{$\begin{array}{l}\text { Lignin } \\
(\mathrm{mg} / \mathrm{g})\end{array}$} & \multirow{2}{*}{ Reference } \\
\hline & Glucose & Xylose & Arabinose & Mannose & & \\
\hline Raw MG & 420 & 190 & 41 & - & 235 & Hideno et al. $2013^{b}$ \\
\hline Raw MS & 360 & 173 & 42 & - & 197 & Hideno et al. $2013^{b}$ \\
\hline Raw RS & 381 & 148 & 140 & - & 148 & Hideno 2017 \\
\hline Raw JC & 449 & 44 & - & 105 & 319 & Hideno et al. 2013a \\
\hline AP70-MG & 751 & 129 & 15 & - & 75.1 & Hideno et al. $2013^{b}$ \\
\hline AP70-MS & 776 & 152 & 17 & - & 10.7 & Hideno et al. $2013^{b}$ \\
\hline AP70-RS & 811 & 86 & N.D. & - & 30.5 & Hideno 2017 \\
\hline APrt-RS & 798 & 70 & N.D. & - & 13.9 & Hideno 2017 \\
\hline AP70-JC & 460 & 16 & - & 16 & 218 & Hideno 2016 \\
\hline
\end{tabular}

The relationships between thermal degradation temperatures of raw samples and the effects of AP treatment are shown in Fig. 2. Both ratio of delignification and cellulose accumulation, which show the effects of AP treatment, severely decreased when subjected to temperatures over $260{ }^{\circ} \mathrm{C}$, corresponding to thermal degradation at $5 \%$ weight loss. The present results show that the samples with lower thermal degradation temperature at an early stage, such as $5 \%$ weight loss, has a susceptibility to AP treatment. Moreover, these results also show that TGA is useful for selection of suitable biomass in the AP treatment. 


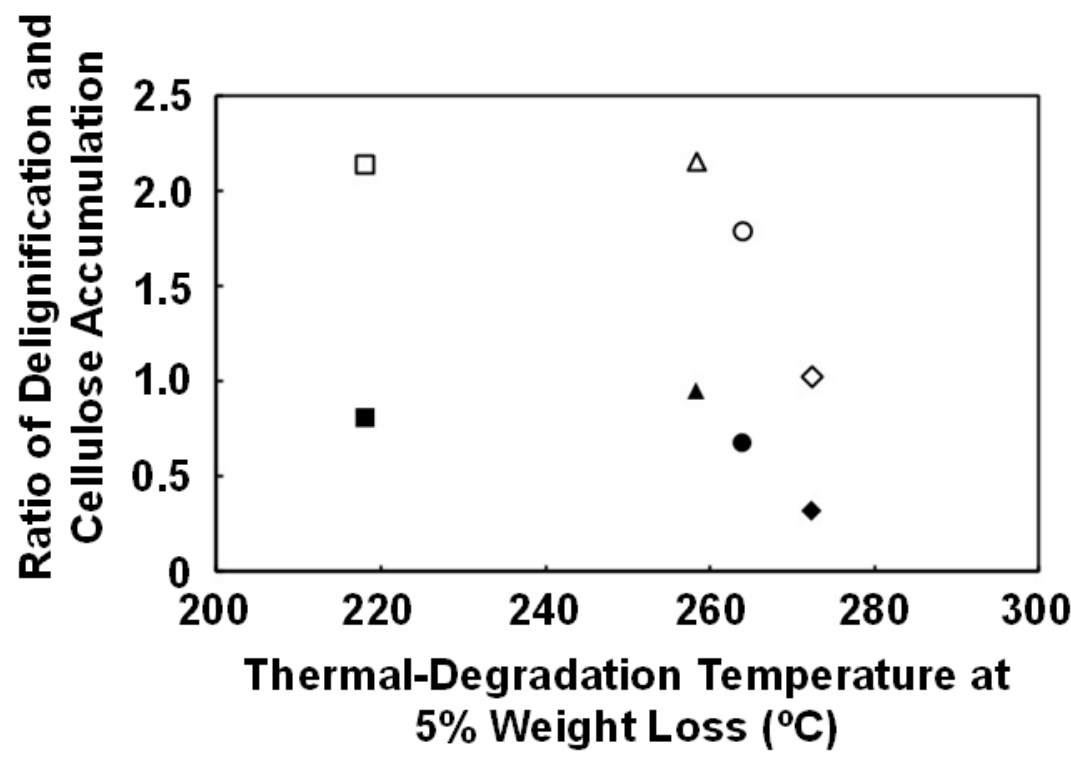

Fig. 2. Relationships between thermal degradation temperature at $5 \%$ weight loss and the ratio of delignification and cellulose accumulation that occurred using AP treatment Closed and open symbols show the ratio of delignification and cellulose accumulation, respectively. Circle, triangle, square, rhombus symbols represent MG, MS, RS, JC, respectively.

The weight loss of $5 \%$ mainly occurs by thermal degradation of a part of hemicellulose and lignin. A previous report suggests that TGA (DTG curve) reflects cellulose components and the denaturing of non-cellulosic substances, such as hemicellulose and lignin (Hideno 2016). Considering the present data and the previous study, the efficiency of AP treatment is greatly affected by the composition and structure of hemicellulose and lignin, which have lower initial thermal degradation temperatures in raw samples.

\section{Comparison of the Reducing Capabilities of Raw and AP treated Biomass}

Kawamoto (2015) suggested that the reducing ends of cellulose strongly affect thermal degradation. In this study, the reducing capability of lignocellulose that comprises cellulose, hemicellulose, and lignin were investigated by a modified BCA method. The reducing capabilities were calculated as the numbers of moles of reducing-end glucose per gram. The reducing capability of untreated biomass was higher than AP treated biomass as shown in Fig. 3. Hemicelluloses such as xylan are significantly decreased using AP treatment in herbaceous biomass like Miscanthus and rice straw (Hideno et al. 2013; Hideno 2016). The author showed that reducing capability is influenced by hemicellulose and the reducing end of cellulose. In addition, thermal decomposition and burnt cellulose deposits are strongly associated with reducing ends; thus, thermal lignocellulose decomposition is significantly correlated with reducing capability derived from hemicellulose and the reducing ends of cellulose. Here, the results showed that the reducing capability was correlated with the components and thermal degradation properties of lignocellulosic materials. Besides, reducing capability varied with hemicellulose content and was associated with lower thermal decomposition temperatures.

The reducing capabilities of the AP treated samples were divided by those for the raw samples. The quotients were 0.086 for $M$. giganteus, 0.056 for $M$. sinensis, 0.063 for rice straw (AP70), 0.089 for rice straw (APrt), and 1.111 for Japanese cypress. These values 
are indicators of AP treatment sensitivity. If the quotient is less than 1.000, the AP treatment will be highly effective on the sample, and if greater than 1.000 the AP treatment will have a low efficiency. These results closely resemble those determined for thermal degradation behavior. The reducing-end glucose units in microcrystalline cellulose strongly affect thermal degradation, dehydration, and carbonization (Kawamoto 2015). Material with high reducing capability is easily oxidized. Therefore, raw MG, MS, and RS with high reducing capabilities, were sensitive to the AP treatment. Considering this and other previous studies, the reducing capability of the lignocellulosic sample also affected thermal degradation behavior and the effectiveness of AP treatment. The ratio of the reducing capability of the raw sample to the AP treated sample is a candidate indicator of AP treatment efficacy.

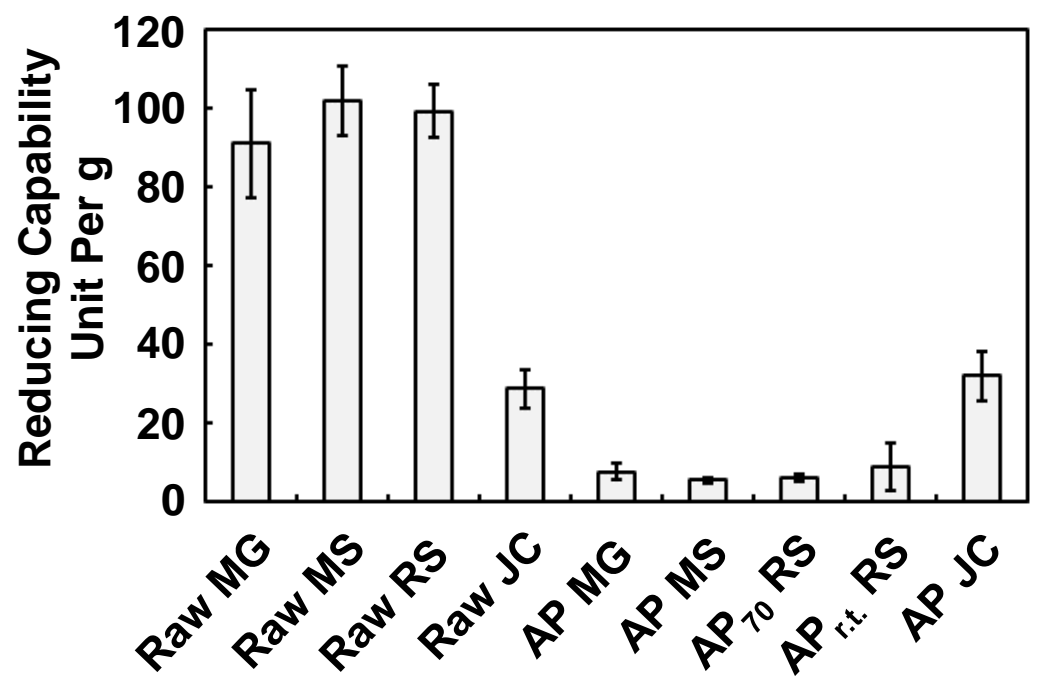

Fig. 3. Comparison of reducing capabilities of raw and alkaline peroxide treated herbaceous biomass; MG, MS, and RS are Miscanthus giganteus, $M$. sinensis, and rice straw, respectively; AP70RS and APr.t.RS represent rice straw subjected to alkaline peroxide treatment at $70{ }^{\circ} \mathrm{C}$ and at room temperature, respectively

\section{Thermal Degradation Property and Crystallinity of AP-treated Rice Straw}

Hideno (2016) proposed that because of chemical compositions and enzymatic digestibility, DTG curves are potential indicators of cellulose purification. TGA was used to characterize the raw and AP treated rice straw samples in this study. Three main peaks and sub-peaks were found in the fitted DTG curves for the RS samples (Fig. 4a). These results corroborate earlier findings (Negro et al. 2003) and (Hideno 2016). The highest peak at approximately $350{ }^{\circ} \mathrm{C}$ may be explained by the thermal decomposition of cellulose. The other two peaks were derived from hemicelluloses. The DTG peaks for the AP treated rice straw samples shifted to higher temperatures and were sharper than those observed for the raw RS samples, as shown in Figs. $4 \mathrm{~b}$ and $4 \mathrm{c}$. The discrete peaks at approximately 350 ${ }^{\circ} \mathrm{C}$ were sharper and higher for the AP treated samples than the raw samples. The results also showed that all the DTG peaks for APrt, including those separated at approximately $350{ }^{\circ} \mathrm{C}$, were slightly sharper and higher than AP70. These results can be explained by the relative differences in the main chemical components of the two samples. The amounts of materials such as hemicellulose and the portion of lignin that underwent thermal degradation of less than $300{ }^{\circ} \mathrm{C}$ were slightly higher in AP70 than APrt. Moreover, the area 
under the separated DTG peaks at approximately $350{ }^{\circ} \mathrm{C}$ were positively correlated with the cellulose content. Therefore, the separated DTG peaks at approximately $350{ }^{\circ} \mathrm{C}$ can estimate cellulose purification; corroborating (Hideno 2016). The results suggest that the APrt can generate pulp with more highly purified cellulose than that obtained from AP70. All XRD spectra of the rice straw samples indicated the presence of cellulose I-type crystals. However, the peaks at 1-10 and 110 did not separate (data not shown). The Segal crystallinity indices (CrI) of raw RS, APrt, and AP70 were 50.4, 69.8, and 68.1\%, respectively. Relative differences in cellulose content may explain that the CrI for APrt was slightly higher than AP70, as shown in Table 1 (Hideno 2017). Alternatively, readsorbed xylan and lignin in AP70 may also account for the slightly lower CrI. Thermal degradation of lignocellulosic biomass occurs on the surface. Hideno et al. (2013a) suggested that high CrI might be correlated with the exposure of crystalline cellulose fibers (microfibrils). Furthermore, this study and indicates that high-crystalline cellulose microfibrils were exposed on the surface of APrt. Therefore, the thermal degradation property of APrt was like the microcrystalline cellulose.

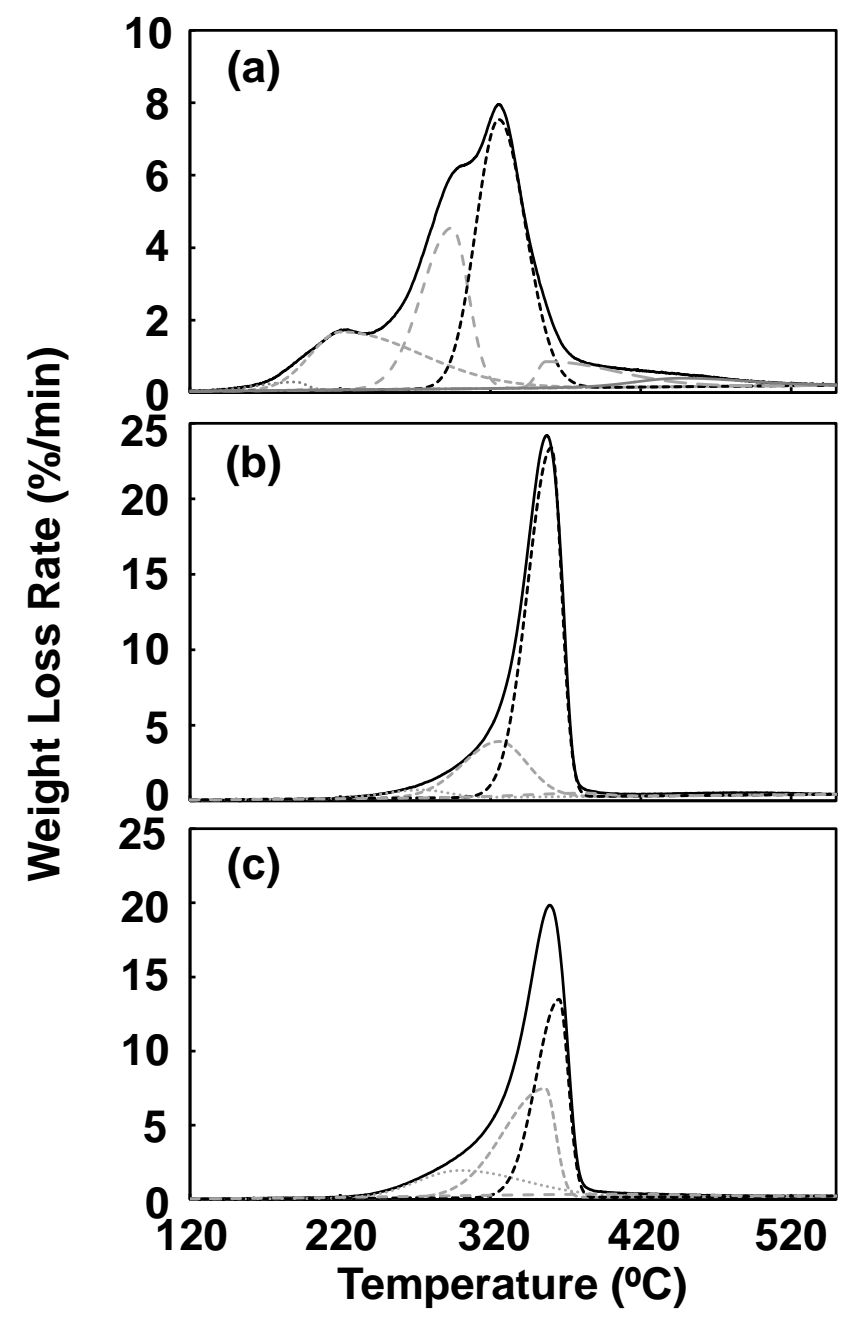

Fig. 4. Derivative thermal gravimetric (DTG) curves (solid lines), and separated and curve-fitted DTG curves (broken lines) of (a) rice straw, (b) rice straw subjected to alkaline peroxide (AP) treatment for $10 \mathrm{~min}$ at approximately $25^{\circ} \mathrm{C}$, and (c) rice straw subjected to AP treatment for greater than $12 \mathrm{~h}$ at $70^{\circ} \mathrm{C}$. 


\section{Morphology of the AP-treated Rice Straw}

Thermal degradation often starts at the surface of materials. Therefore, the surface morphology of rice straw was investigated by FE-SEM (Fig. 5) before and after AP treatment. Fibers were not readily distinguishable on the surface of raw RS but the matrix was clearly visible. The initial temperature of the thermal decomposition of raw RS was low. The matrix most likely contained hemicellulose and lignin, which have lower initial thermal degradation temperatures than cellulose. Microfibrils were observed on the surface of the AP treated rice straw. Based on the results of the constituent sugar analysis, they comprise crystalline cellulose (Hideno 2017). The DTG curve of AP treated rice straw (Fig. 1) matched those of microcrystalline cellulose (Hideno, 2016) and the XRD spectrum. Cellulase molecules easily attacked the exposed cellulose microfibrils and penetrated the AP-treated rice straw samples through nano-scale pores. Therefore, sponge-like pulp fibers could be obtained from short-duration, room-temperature (approximately $25{ }^{\circ} \mathrm{C}$ ) AP treatment. This configuration improves enzymatic hydrolysis, and the effects are similar to those of thermal degradation conditions. Morphological changes caused by AP treatment could enhance enzymatic digestibility (Hideno 2017). The effects of AP treatment on microcrystalline cellulose may be highly homologous to those of thermal degradation. Enzymatic digestibility and thermal degradation in AP-treated rice straw resemble those for lignocellulosic samples subjected to other treatments, such as hot-compressed water and organosolv solvents (Hideno et al. 2009, 2013a,b, 2016). All these treatments produced similar major decreases in lignin and hemicellulose content, and higher crystallinity and enzymatic digestibility. The treatments also require similar enzyme levels to achieve high digestibility.
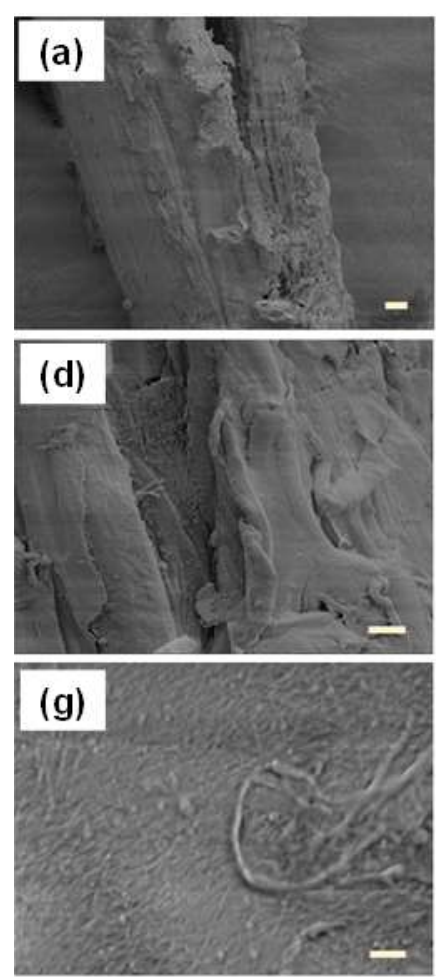
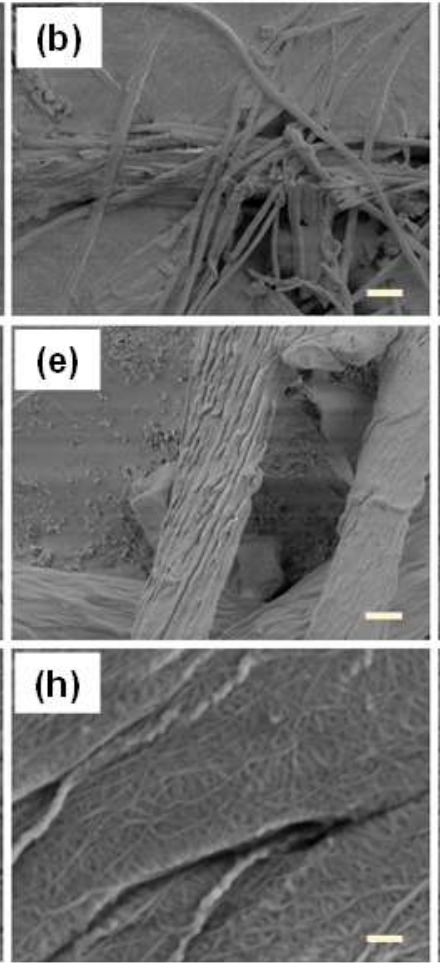
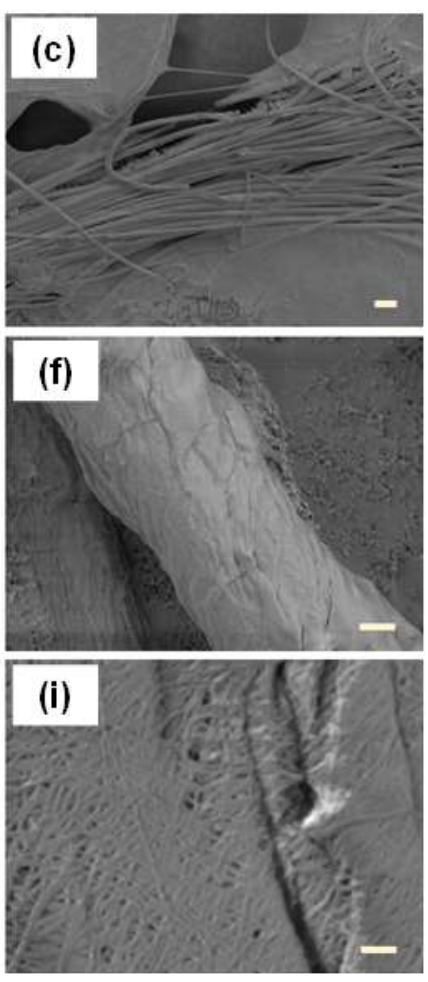

Fig. 5. Field-emission type scanning electron micrographs of untreated rice straw (a, d, and g), alkaline peroxide (AP)-treated rice straw at approximately $25^{\circ} \mathrm{C}(\mathrm{b}, \mathrm{e}$, and $\mathrm{h})$, and AP-treated rice straw at $70{ }^{\circ} \mathrm{C}(\mathrm{c}, \mathrm{f}$, and i). Scale bars of (a)-(c), (d)-(f), and (g)-(i) represent $20 \mu \mathrm{m}, 2 \mu \mathrm{m}$, and $200 \mathrm{~nm}$, respectively. 


\section{CONCLUSIONS}

1. Raw rice straw was the most suitable material for short-duration AP treatment. Among the four lignocellulosic samples, it had the lowest peak thermal degradation temperature at $1 \%$ weight loss. The thermal degradation of $1 \%$ weight loss for raw RS occurred at $180.6{ }^{\circ} \mathrm{C}$, raw $\mathrm{MS}$ at $209.6{ }^{\circ} \mathrm{C}$, raw MG at $225.5^{\circ} \mathrm{C}$, and raw JC at 232.3 ${ }^{\circ} \mathrm{C}$.

2. In AP-treated samples, rice straw had the highest thermal degradation temperature. The thermal degradation of DTG peak occurred for AP RS at $359.2^{\circ} \mathrm{C}$, AP MS at $343.5^{\circ} \mathrm{C}$, AP MG at $334.8^{\circ} \mathrm{C}$, and AP JC at $313.3^{\circ} \mathrm{C}$.

3. The rice straw and $M$. sinensis with a thermal degradation temperature less than 260 ${ }^{\circ} \mathrm{C}$ at $5 \%$ weight loss resulted in a higher ratio of delignification and cellulose accumulation in AP treatment.

4. When the reducing capabilities of the AP treated samples were divided by those for the raw samples, the quotients were 0.086 for $M$. giganteus, 0.056 for $M$. sinensis, 0.063 for rice straw (AP70), 0.089 for rice straw (APrt), and 1.111 for Japanese cypress. These values are related to thermal degradation behaviors and are indicators of AP treatment sensitivity.

5. According to the DTG curves, XRD spectra, and FE-SEM observations, crystalline cellulose nanofibers were exposed on the surface of AP-treated rice straw.

\section{ACKNOWLEDGMENTS}

The author thanks the Advanced Research Support Center of Ehime University for leasing instruments for TGA, FE-SEM, and XRD analyses. The author would also like to thank Prof. Hiroyuki Yano (Kyoto University, Uji, Japan) for the valuable comments, Dr. Yo Toma (Ehime University, Matsuyama, Japan), and Prof. Toshihiko Yamada (Hokkaido University, Sapporo, Japan) for kindly supplying rice straw and Miscanthus plants, respectively. This work was supported by JSPS KAKENHI (Grant No. JP26850222 and JP16K07809).

\section{REFERENCES CITED}

Antal Jr., M. J., and Varhegyi, G. (1995). "Cellulose pyrolysis kinetics: The current state of knowledge," Ind. Eng. Chem. Res. 34, 703-717. DOI: 10.1021/ie00042a001

Burhenne, L., Messmer, J., Aicher, T., and Laborie, M. P. (2013). "The effect of the biomass components lignin, cellulose, and hemicellulose on TGA and fixed bed pyrolysis," J. Anal. Appl. Pyro. 101, 177-184. DOI: 10.1016/j.jaap.2013.01.012

Hendriks, A. T. M., and Zeeman, G. (2008). "Pretreatments to enhance the digestibility of lignocellulosic biomass," Bioresour. Technol. 100(1), 10-18.

DOI: 10.1016/j.biortech.2008.05.027

Hideno, A. (2016). "Comparison of the thermal degradation properties of crystalline and amorphous cellulose, as well as treated lignocellulosic biomass," BioResources 11(3), 6309-6319. DOI: 10.15376/biores.11.3.6309-6319 
Hideno, A. (2017). "Short-time alkaline peroxide pretreatment for rapid pulping and efficient enzymatic hydrolysis of rice straw," Bioresour. Technol. 230, 140-142. DOI: 10.1016/j.biortech.2017.01.058

Hideno, A., Abe, K., and Yano, H. (2014) "Preparation using pectinase and characterization of nanofibers from orange peel waste in juice factories," J. Food Sci. 79(6), N1218-N1224. DOI: 10.1111/1750-3841.12471

Hideno, A., Inoue, H., Tsukahara, K., Fujimoto, S., Minowa, T., Inoue, S., Endo, T., and Sawayama, S. (2009). "Wet disk milling pretreatment without sulfuric acid for enzymatic hydrolysis of rice straw," Bioresour. Technol. 100(10), 2706-2711. DOI: 10.1016/j.biortech.2008.12.057

Hideno, A., Inoue, H., Tsukahara, K., Yano, S., Fang, X., Endo, T., and Sawayama, S. (2011). "Production and characterization of cellulases and hemicellulases by Acremonium cellulolyticus using rice straw subjected to various pretreatments as the carbon source," Enz. Microbial. Technol. 48(2), 162-168.

DOI: 10.1016/j.enzmictec.2010.10.005

Hideno, A., Kawashima, A., Endo, T., Honda, K., and Morita, M. (2013a). "Ethanolbased organosolv treatment with trace hydrochloric acid improves the enzymatic digestibility of Japanese cypress (Chamaecyparis obtusa) by exposing nanofibers on the surface," Bioresour. Technol. 132, 64-70. DOI: 10.1016/j.biortech.2013.01.048

Hideno, A., Kawashima, A., Anzoua, K. G., and Yamada, T. (2013b). "Comparison of the enzymatic digestibility of physically pretreated selected line of diploidMiscanthus sinensis Shiozuka and triploid-M.×giganteus," Bioresour. Technol. 146, 393-399. DOI: 10.1016/j.biortech.2013.07.084

Hilbers, T. J., Wang, Z., Pecha, B., Westerhof, R. J. M., Kersten, S. R. A., PelaezSamaniego, M. R. and Garcia-Perez, M. (2015). "Cellulose-lignin interactions during slow and fast pyrolysis," J. Anal. Appl. Pyro. 114, 197-207.

DOI: $10.1016 /$ j.jaap.2015.05.020

Hon, D. N. S. (1994). “Cellulose: A random walk along its historical path,” Cellulose 1, $1-25$.

Isogai, A., Saito, T., and Fukuzumi, H. (2011). "TEMPO-oxidized cellulose nanofibers," Nanoscale 3(1), 71-85. DOI: 10.1039/C0NR00583E

Kawamoto, H. (2015). "Reactions and molecular mechanisms of cellulose pyrolysis," $J$. Jap. Wood Res. Soc. 61(1), 1-24. DOI: 10.2488/jwrs.61.1

Kim, S., and Dale, B. E. (2004). "Global potential bioethanol production from wasted crops and crop residues," Biomass Bioenerg. 26(4), 361-375.

DOI: 10.1016/j.biombioe.2003.08.002

Kleppe, P. J. (1970). “Kraft pulping,” TAPPI J. 53(1), 35-47.

Mosier, N., Wyman, C., Dale, B., Elander, R., Lee, Y. Y., Holtzapple, M., and Ladisch, M. (2005). "Features of promising technologies for pretreatment of lignocellulosic biomass," Bioresour. Technol. 96(6), 673-686. DOI: 10.1016/j.biortech.2004.06.025

Negro, M. J., Manzanares, P., Oliva, J. M., Ballesteros, I., and Ballesteros, M. (2003). "Changes in various physical/chemical parameters of Pinus pinaster wood after steam explosion pretreatment," Biomass Bioenerg. 25(3), 301-308. DOI: 10.1016/S09619534(03)00017-5

Uetani, K., Watanabe, Y., Abe, K., and Yano, H. (2014). "Influence of drying method and precipitated salts on pyrolysis for nanocelluloses," Cellulose 21(3), 1631-1639. DOI: $10.1007 / \mathrm{s} 10570-014-0242-2$ 
Yano, H. (2010). "Production of cellulose nanofibers and their application," Funct. Pap. Res. J. 49, 15-20. DOI: 10.11332/kinoushi.49.15

Zhang, Y. H. P., and Lynd, L. R. (2005). "Determination of the number-average degree of polymerization of cellodextrins and cellulose with application to enzymatic hydrolysis," Biomacromolecules 6(3), 1510. DOI: 10.1021/bm049235j

Article submitted: April 17, 2018; Peer review completed: July 7, 2018; Revised version received and accepted: June 24, 2020; Published: July 1, 2020.

DOI: 10.15376/biores.15.3.6217-6229 